\title{
Système de classification du confort et de la sécurité des voies cyclables canadiennes (Can-BICS) : convention d'appellation commune des aménagements cyclables
}

\author{
Meghan Winters, Ph. D. (1); Moreno Zanotto, M. Sc. (1); Gregory Butler, M. Sc. (2)
}

Diffuser cet article sur Twitter

\begin{abstract}
Résumé
Il n'existe pas de convention d'appellation pour les aménagements cyclables valable pour toutes les villes. Notre but était d'établir une nomenclature commune pour les aménagements cyclables au Canada qui soit pertinente en contexte de santé publique. Nous avons examiné plusieurs guides de conception technique des transports ainsi que des directives en santé publique pour mettre au point un système de classification des aménagements cyclables, soit le Système de classification du confort et de la sécurité des voies cyclables canadiennes (ou Can-BICS, de l'anglais Canadian Bikeway Comfort and Safety). Ce système à trois niveaux répartit les aménagements cyclables en cinq catégories, selon leur degré de sécurité et leur confort pour les usagers. Adopter une nomenclature uniforme comme le système Can-BICS va contribuer aux mesures de surveillance régionale et nationale en santé publique, à la planification et à la durabilité.
\end{abstract}

Mots-clés : données ouvertes, transport actif, cyclisme, infrastructure, nomenclature

\section{Introduction}

L'augmentation du nombre de cyclistes et de déplacements en vélo est un objectif commun aux programmes de santé publique, de durabilité et de transport ${ }^{1-4}$. Malgré le fait que de nombreuses villes collectent des données sur leurs aménagements cyclables et, de plus en plus, publient ces données dans le cadre d'initiatives de données ouvertes, il n'existe pas de convention d'appellation pour décrire les aménagements cyclables. Cette absence de nomenclature nuit aux recherches et aux pratiques visant à éclaircir le rôle des aménagements cyclables dans le transport actif au sein des collectivités.

Notre but consistait à établir une nomenclature pour les aménagements cyclables au Canada qui soit pertinente en santé publique. Cette nomenclature constitue une étape fondamentale vers l'opérationnalisation d'indicateurs utilisables dans les activités de recherche et de surveillance en santé publique portant sur l'activité physique au Canada ${ }^{5}$. Nous avons pris en compte les aménagements cyclables euxmêmes, soit les pistes et les voies destinées aux vélos, mais non les installations pour navetteurs actifs comme les aires de stationnement pour vélos, les vestiaires ou les douches, qui ne font pas forcément l'objet d'un suivi.

Cette étude est exemptée de l'examen par le Comité d'éthique de la recherche, car elle s'appuie uniquement sur des données publiques pour lesquelles il n'y a aucune attente raisonnable en matière de protection de la vie privée.

\section{Méthodologie}

Survol

Nous avons examiné plusieurs guides de conception technique des transports et

\section{Points saillants}

- Il est nécessaire d'établir une nomenclature commune pour les aménagements cyclables au Canada afin d'améliorer les mesures de surveillance en santé publique en matière de milieux de transport actif.

- Le système Can-BICS est un système de classification des aménagements cyclables à trois niveaux qui définit le degré de sécurité et le confort pour les usagers de cinq types d'aménagement cyclable.

- Les voies cyclables très confortables sont peu stressantes : ce sont principalement les pistes cyclables sur chaussée longeant les rues principales, les voies cyclables dans les rues secondaires ("vélorues») et les pistes en site propre.

- Les voies cyclables moyennement confortables sont peu ou moyennement stressantes : ce sont principalement les sentiers polyvalents longeant une chaussée ou formant un corridor indépendant.

- Les voies cyclables peu confortables sont très stressantes : ce sont principalement les bandes cyclables peintes au sol sur des routes achalandées.

plusieurs directives en santé publique afin de mettre au point un système de classification fondé sur le degré de sécurité (risque de blessure ou d'accident selon le type d'aménagement) et le confort pour les usagers (préférences en matière

Rattachement des auteurs :

1. Faculté des sciences de la santé, Université Simon Fraser, Burnaby (Colombie-Britannique), Canada

2. Agence de la santé publique du Canada, Ottawa (Ontario), Canada

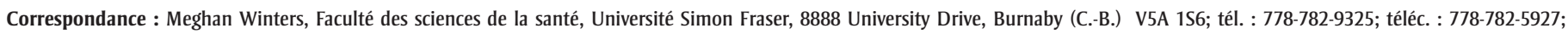
courriel : mwinters@sfu.ca 
d'aménagement sur les plans du confort et du stress). Nous avons également compilé les termes d'aménagement utilisés dans les données ouvertes fournies par les municipalités canadiennes et nous les avons intégrés au système de classification de la nomenclature.

\section{Examen des guides de conception technique}

Nous avons examiné les guides de conception technique du Canada et des ÉtatsUnis publiés au cours des 5 dernières années pour déterminer comment les aménagements cyclables étaient définis et classés. Cet examen nous a orienté vers d'autres documents pertinents. Nous avons ainsi examiné les documents suivants : Guide canadien de conception géométrique des routes ${ }^{6}$, de l'Association des transports du Canada (ATC), Transportation design guidelines: all ages and abilities cycling route, de la ville de Vancouver ${ }^{7}$, Design manual for bicycle traffic, de la plateforme $\mathrm{CROW}^{8}$, Urban bikeway design guide ${ }^{9}$ et Designing for all ages and abilities: Contextual guidance for high-comfort bicycle facilities ${ }^{10}$, de l'Association nationale des responsables des transports urbains (NACTO), et enfin Separated bike lane planning and design guide ${ }^{11}$, du département des Transports du Massachusetts.

\section{Mise au point de la classification}

Pour orienter notre projet de nomenclature, nous avons analysé, dans chacun des guides techniques, les méthodes de classification employées et leur justification. Nous avons aussi examiné la littérature en santé publique sur la sécurité et les préférences en matière d'aménagement, car la sécurité et le confort forment les principes de base de l'organisation des structures de classification (pour plus de détails, veuillez consulter l'analyse exposée dans le rapport The Canadian Bikeway Comfort and Safety (Can-BICS) classification system: A proposal for developing common naming conventions for cycling infrastructure ${ }^{12}$ ). Notre système de classification préliminaire a été revu par un spécialiste américain et trois spécialistes canadiens, soit deux en santé publique et deux en planification et en conception des transports. Leur rétroaction a entraîné une modification des définitions des aménagements, mais aucun changement notable à la classification.

\section{Analyse des données ouvertes}

Notre but était de caractériser l'éventail des termes qualifiant les aménagements cyclables utilisés par les collectivités canadiennes afin de saisir la portée de la nomenclature et son degré de correspondance avec le système Can-BICS proposé. Pour former un échantillon national, nous avons choisi la tranche des $10 \%$ des subdivisions de recensement les plus peuplées de chaque province et territoire. Mises ensemble, ces 45 subdivisions de recensement sont représentatives de 50,4\% de la population canadienne.

Nous avons constitué un ensemble de données sur les aménagements cyclables en regroupant les données ouvertes et des cartes des réseaux cyclables municipaux au besoin. Nous avons extrait tous les termes mentionnés dans les données municipales et nous les avons répartis selon le système de classification Can-BICS. Lorsque c'était possible (pour environ $60 \%$ des termes), nous avons associé l'appellation de l'aménagement à l'un des cinq aménagements cyclables du système Can-BICS en se fiant uniquement à son appellation (p. ex. les « voies cyclables» ont été classées comme "bandes cyclables »). Pour assurer la rigueur du projet, nous avons mené des vérifications ponctuelles sur $10 \%$ des termes d'aménagement en situant et en identifiant les aménagements à l'aide de Google Street View et de QGIS : nous n'avons relevé aucune discordance. Dans les cas où le terme ne permettait pas de classer facilement l'aménagement (dans environ $40 \%$ des cas), nous avons utilisé Google Street View (un service d'imagerie en ligne pour visualiser les rues) et QGIS (un système d'information géographique). Les fichiers de données ouvertes et les données sur les termes d'aménagement sont accessibles dans le dépôt de données RADAR de l'Université Simon Fraser (researchdata .sfu.ca).

\section{Résultats}

Les six guides de conception technique décrivaient de nombreux aménagements cyclables. Nous les avons regroupés en cinq catégories («bandes cyclables peintes au sol ", " vélorues ", " pistes cyclables sur chaussée », " pistes cyclables en site propre " et "sentiers polyvalents ») en fonction de leur conception (design), de leur utilisation exclusive ou non par les cyclistes et de la proximité entre les cyclistes et les autres usagers de la route ${ }^{6-7}$. Plusieurs guides ont traité de la sécurité (p. ex. séparation par rapport aux véhicules motorisés), mais les préférences des usagers et le confort ont été peu abordés, particulièrement dans le cas des aménagements cyclables le long des routes.

L'intégration des guides de pratique et des considérations liées à la santé publique pour classer les aménagements susceptibles de favoriser le plus les déplacements et la sécurité à vélo nous a permis de mettre au point un système de classification du confort et de la sécurité des voies cyclables canadiennes (Can-BICS). Ce système de classification à trois niveaux répartit les aménagements cyclables en cinq catégories, selon leur degré de sécurité et leur confort pour les usagers (tableau 1) :

- Voies cyclables très confortables. Ces voies cyclables peu stressantes sont confortables pour la plupart des usagers. Il s'agit des pistes cyclables sur chaussée longeant les voies principales, des vélorues et des pistes cyclables en site propre.

- Voies cyclables moyennement confortables. Ces voies cyclables peu à moyennement stressantes sont jugées confortables par une partie des usagers. Les sentiers polyvalents hors route appartiennent à cette catégorie. Ces sentiers polyvalents, qui sont partagés avec les piétons et les usagers d'autres modes de transport actif, sont situés le long d'une chaussée ou dans un corridor indépendant.

- Voies cyclables peu confortables. Ces aménagements cyclables très stressants sont confortables pour peu d'usagers. L'aménagement typique de cette catégorie est une bande cyclable peinte au sol, qui incite les cyclistes à circuler sur des bandes peintes sur le sol de routes achalandées.

\section{Comparaison des termes d'aménagement dans les données ouvertes au système Can-BICS}

Sur les 45 municipalités retenues, $89 \%$ ( $n=40 / 45$ ) avaient un catalogue de données ouvertes et $80 \%$ parmi ces dernières ( $n=32 / 40$ ) disposaient d'un ensemble de données sur les aménagements cyclables. Ces données ont été publiées entre 2005 et 2019. Nous avons extrait 269 termes d'aménagement cyclable de ces données (entre 2 et 14 selon les subdivisions de recensement), après élimination des aménagements piétonniers évidents 
TABLEAU 1

Système de classification du confort et de la sécurité des voies cyclables canadiennes (Can-BICS)

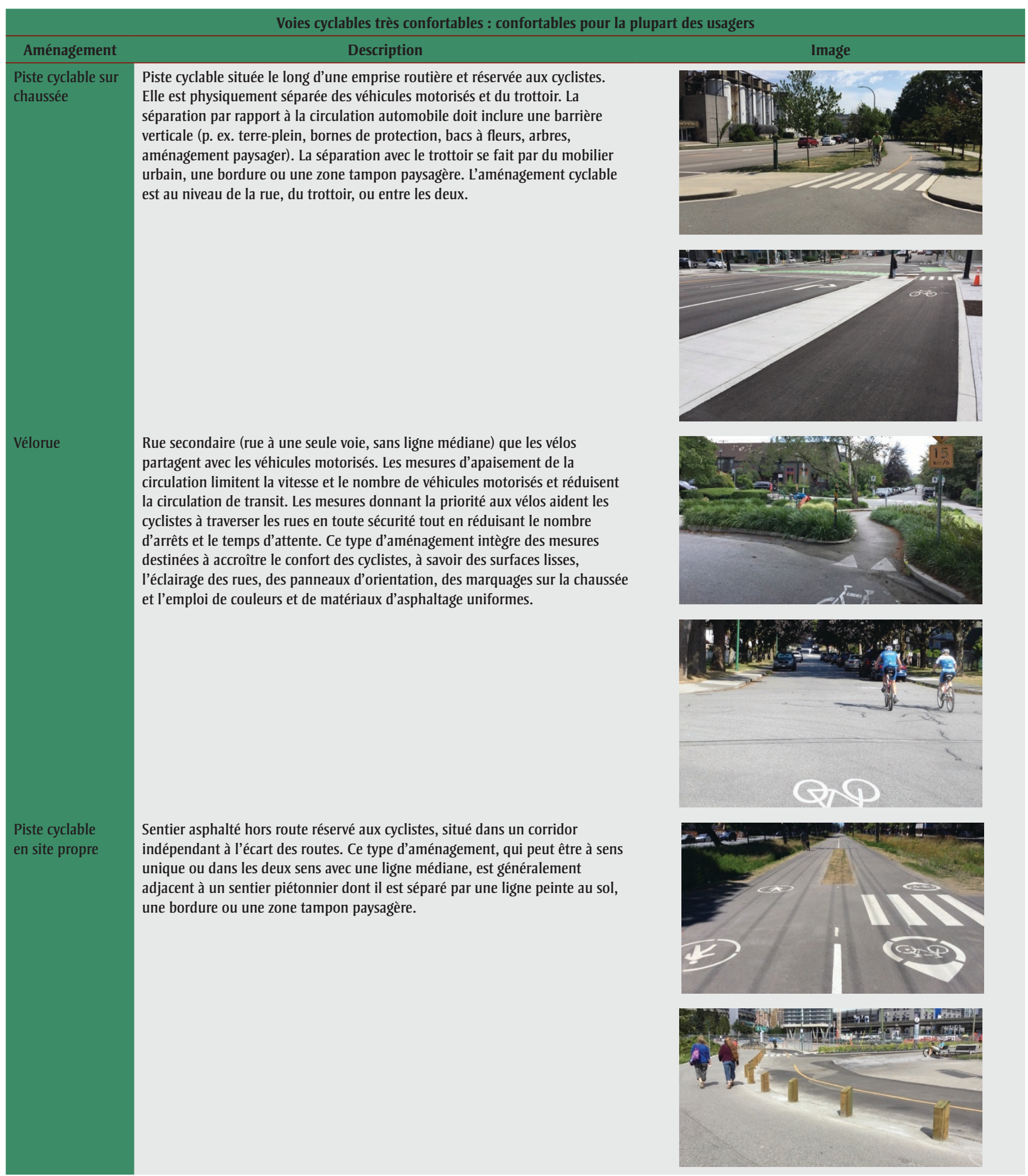

Suite à la page suivante 


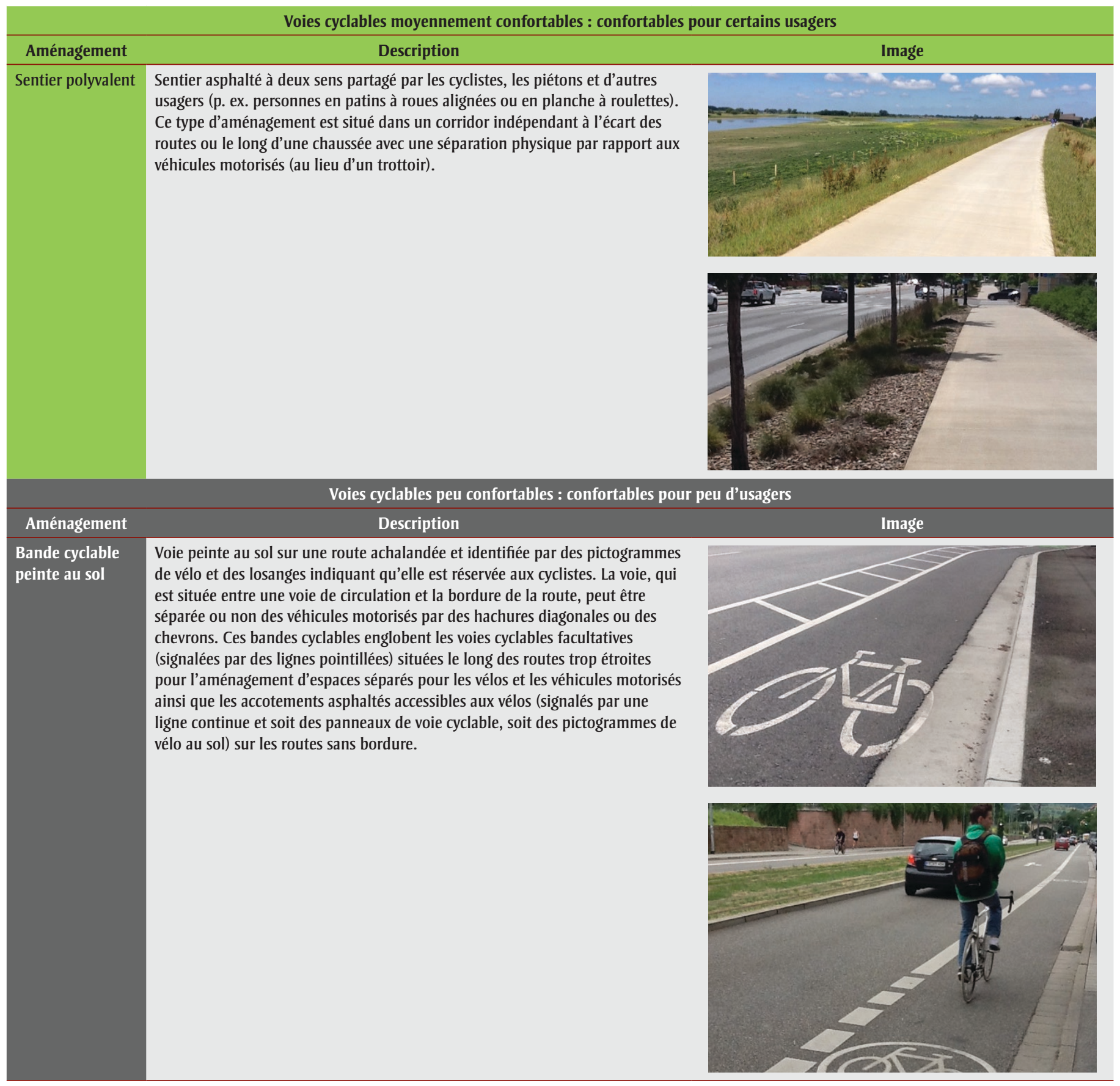

Source : The Canadian Bikeway Comfort and Safety (Can-BICS) Classification System: A Proposal for Developing Common Naming Conventions for Cycling Infrastructure ${ }^{12}$.

(p. ex. escaliers, trottoirs), des segments de route et des routes désaffectées. Environ 100 termes différents sont demeurés, après regroupement des termes apparentés (p. ex. "bike lane » et " bicycle lane »). Nous avons associé $60 \%$ des 269 termes à l'un des cinq types d'aménagement cyclable du système Can-BICS en fonction de l'appellation seulement (p. ex. bandes cyclables classées comme bandes cyclables peintes au sol). Nous avons évalué les $40 \%$ restants $(n=108)$ à l'aide de Google Street View (voir The Canadian
Bikeway Comfort and Safety (Can-BICS) classification system: A proposal for developing common naming conventions for cycling infrastructure) ${ }^{12}$.

Nous avons comparé la nomenclature des données ouvertes municipales à celle du système Can-BICS (figure 1) pour vérifier le degré de chevauchement. Il importe de souligner que les proportions présentées dans cet article correspondent à la fréquence d'utilisation des termes qualifiant l'aménagement cyclable dans les fichiers de données ouvertes et non à la part proportionnelle de ce type d'aménagement au sein du réseau cyclable. Nous avons constaté que $23 \%$ des termes présents dans les données ouvertes correspondaient à des voies cyclables très confortables : $8 \%$ étaient des pistes cyclables sur chaussée, $12 \%$ étaient des vélorues et $3 \%$ étaient des pistes cyclables en site propre. Dans l'ensemble, $24 \%$ des voies cyclables étaient moyennement confortables (sentiers polyvalents) et $28 \%$ étaient peu confortables (bandes cyclables peintes au sol). 
FIGURE 1

Répartition, dans le système de classification Can-BICS, des termes d'aménagement cyclable mentionnés dans les données ouvertes municipales ${ }^{a}$

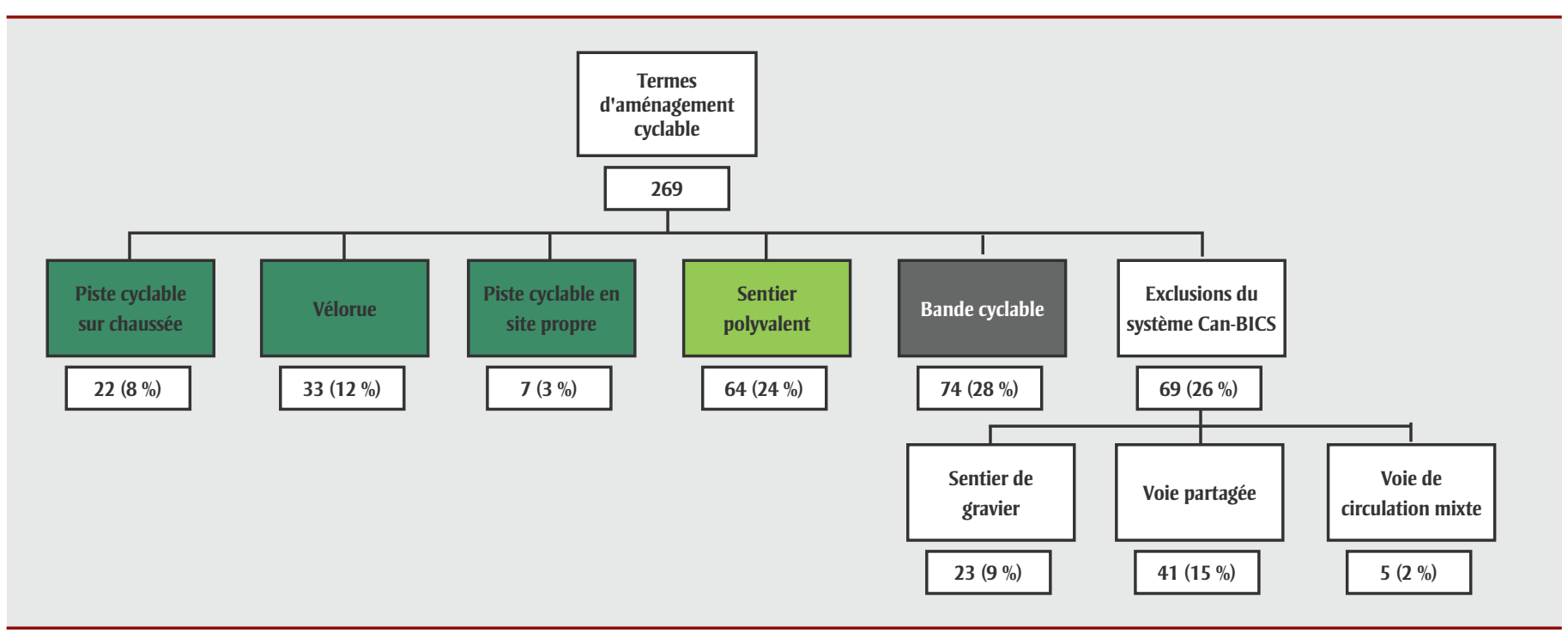

Source : The Canadian Bikeway Comfort and Safety (Can-BICS) Classification System: A Proposal for Developing Common Naming Conventions for Cycling Infrastructure ${ }^{12}$.

Abréviation : Can-BICS, Système de classification de la commodité et de la sécurité des voies cyclables canadiennes.

a 269 termes d'aménagement recensés dans 45 subdivisions de recensement.

Par ailleurs, certains termes d'aménagement mentionnés dans les données ouvertes ne correspondaient pas aux critères du système Can-BICS : on les a considérés, d'après l'état actuel des connaissances, comme n'étant pas adaptés (c.-à-d. non sécuritaires ou non confortables) à la promotion du vélo auprès de la population de tous les âges et de toutes les aptitudes. Un grand nombre de ces aménagements étaient des voies partagées, c'est-à-dire des marquages sur une voie destinée aux automobiles. Or il n'y a pas de preuve que les marquages augmentent la sécurité, et la majorité des cyclistes ne souhaitent pas partager une voie de circulation avec des véhicules motorisés. Les autres aménagements exclus ont été les sentiers de gravier, notamment les sentiers polyvalents dont la surface est en gravier, en terre ou en granulat, les sentiers de vélo de montagne, les sentiers de marche dans un parc et les sentiers de randonnée pédestre en terre battue. Les directives de l'Association des transports du Canada excluent explicitement les sentiers de gravier, avec l'argument qu'ils ne sont accessibles qu'à un faible nombre de vélos et qu'ils présentent des exigences de conception particulières $^{6}$. Enfin, si le réseau de " circulation mixte " (rues ou routes secondaires non améliorées) est susceptible de servir de liaison avec le réseau cyclable principal, il ne comporte aucune signalisation ni mesure d'apaisement de la circulation et ne constitue donc pas un aménagement cyclable. Ces trois catégories rassemblaient $26 \%$ des termes d'aménagement recensés dans les données ouvertes.

\section{Analyse}

Le Système de classification du confort et de la sécurité des voies cyclables canadiennes (Can-BICS) définit cinq types d'aménagement cyclable, répartis en trois niveaux selon leur degré de sécurité et leur confort pour les usagers. La classification s'appuie sur un examen des guides de pratique professionnelle concernant la conception des aménagements cyclables, sur la littérature en santé publique en matière de sécurité et de préférences des usagers et sur une analyse des conventions d'appellation en usage. Cette approche met l'accent sur les aménagements cyclables les plus sécuritaires et qui sont préférés des personnes de tous les âges et de toutes les aptitudes, conformément à l'objectif en santé publique d'augmenter le nombre de cyclistes et de déplacements en vélo et, du même coup, le bien-être individuel et collectif ${ }^{13}$.

Il est essentiel d'établir une nomenclature normalisée pour les mesures de surveillance en santé publique, car cela permettra de comparer l'accessibilité et la nature des aménagements dans différents milieux et au fil du temps. Nous nous attendons à ce que les planificateurs puissent utiliser la nomenclature normalisée du système
Can-BICS pour classer les voies de leur collectivité et pour produire des données spatiales détaillées et des indicateurs utiles aux autorités locales en santé publique.

En ce qui concerne le système Can-BICS, les prochaines étapes consisteront à opérationnaliser les indicateurs (p. ex. nombre de kilomètres de voies très confortables, moyennement confortables et peu confortables par région), à définir des unités spatiales (p. ex. aire de diffusion) et leurs limites ainsi qu’à évaluer la qualité des sources de données ouvertes. De nouveaux travaux indiquent qu'OpenStreetMap (OSM; openstreetmap.org) est une source de données prometteuse pour les villes canadiennes $^{14}$. À l'échelle nationale, il faut évaluer l'accessibilité, l'exhaustivité et la comparabilité de toutes les sources de données, mais le potentiel de ces efforts de rationalisation et de normalisation est fort.

\section{Points forts et limites}

La conception (design) du réseau est essentielle. Bien que le système Can-BICS s'appuie sur des définitions génériques du confort et de la sécurité, il existe des nuances. Par exemple, une piste cyclable sur chaussée (voie très confortable) mal conçue pourrait comporter un plus grand risque de blessure qu'une bande cyclable peinte au sol (voie peu confortable) bien 
conçue. Le traitement des intersections et la connectivité du réseau ont également une influence sur la sécurité et le confort de circulation.

\section{Conclusion}

Peu de travaux ont été menés jusqu’à présent pour uniformiser l'appellation des infrastructures cyclables des villes. Pourtant, cette entreprise est importante si l'on veut pouvoir comparer des villes (ou les quartiers d'une ville) dans le cadre d'une approche nationale $\mathrm{e}^{15,16}$. Une nomenclature normalisée comme celle du système Can-BICS constitue une étape fondamentale vers l'établissement d'une capacité de surveillance des environnements cyclables urbains par la santé publique.

\section{Remerciements}

Cette étude a été financée par l'Agence de la santé publique du Canada $\left(\mathrm{n}^{\circ}\right.$ 4500387514). Meghan Winters est soutenue par une bourse du Scholar Program de la Michael Smith Foundation for Health Research.

Nous souhaitons remercier les gouvernements municipaux qui ont mis leurs données ouvertes à notre disposition. Nous souhaitons également remercier les quatre spécialistes de la santé publique et de l'ingénierie de la circulation qui ont fourni leur rétroaction sur les versions antérieures du système de classification et les deux personnes qui ont aidé pour les traductions du français.

\section{Contributions des auteurs et avis}

MW a dirigé la conceptualisation du projet, supervisé l'analyse et contribué à la rédaction et la révision du manuscrit. $\mathrm{MZ}$ a dirigé la revue de la littérature, la collecte de données et l'analyse des données et a contribué à la rédaction et la révision du manuscrit. GB a contribué à la conceptualisation du projet et à la rédaction et la révision du manuscrit.

Le contenu de l'article et les points de vue qui y sont exprimés n'engagent que les auteurs et ne correspondent pas nécessairement à ceux du gouvernement du Canada.

\section{Références}

1. Pucher J, Buehler R. City cycling. Cambridge (MA): MIT Press; 2012. doi:10.7551/mitpress/9434.001.0001.
2. Winters $\mathrm{M}$, Teschke $\mathrm{K}$, Grant $\mathrm{M}$, Setton EM, Brauer M. How far out of the way will we travel? Transp Res Rec. 2010;2190(1):1-10. doi:10.3141 /2190-01.

3. Winters M, Teschke K. Route preferences among adults in the near market for bicycling: findings of the Cycling in Cities Study. Am J Health Promot. 2010;25(1):40-47. doi:10.4278 /ajhp.081006-QUAN-236.

4. Teschke K, Harris MA, Reynolds CC, et al. Route infrastructure and the risk of injuries to bicyclists: a case-crossover study. Am J Public Health. 2012;102(12):2336-2343. doi: 10.2105/AJPH.2012.300762.

5. Roberts KC, Butler G, Branchard B, et al. Cadre d'indicateurs de l'activité physique, du comportement sédentaire et du sommeil (APCSS). Promotion de la santé et prévention des maladies chroniques au Canada. 2017; 37(8):276-280. doi:10.24095/hpcdp .37.8.04f.

6. Association des transports du Canada. Chapitre 5 - Conception intégrée pour cyclistes. Dans : Guide canadien de conception géométrique des routes. Ottawa (Ont.) : Association des transports du Canada; 2017.

7. City of Vancouver. Transportation design guidelines: all ages and abilities cycling routes. Vancouver (BC): City of Vancouver; 2017. En ligne à : https://vancouver.ca/files/cov/design -guidelines-for-all-ages-and-abilities -cycling-routes.pdf

8. Platform CROW. Design manual for bicycle traffic. Ede (Netherlands): CROW; 2016.

9. National Association of City Transportation Officials. Urban bikeway design guide, 2nd ed. Washington (DC): NACTO; 2014.

10. National Association of City Transportation Officials. Designing for all ages \& abilities: contextual guidance for high-comfort bicycle facilities. Washington (DC): NACTO; 2017. En ligne à : https://nacto.org/publication /urban-bikeway-design-guide/designing -ages-abilities-new/
11. Massachusetts Department of Transportation. Separated bike lane planning \& design guide [Internet]. Boston (MA): MassDOT; 2015 [consultation le 4 décembre 2017]. En ligne à : https://www.mass.gov/lists/separated -bike-lane-planning-design-guide

12. Winters M, Zanotto $\mathrm{M}$. The Canadian Bikeway Comfort and Safety (CanBICS) Classification System: a proposal for developing common naming conventions for cycling infrastructure. Vancouver (BC): CHATR; 2019. En ligne à : https://chatrlab.ca/projects/

13. Schepers P, Twisk D, Fishman E, Fyhri A, Jensen A. The Dutch road to a high level of cycling safety. Saf Sci. 2017;92:264-73. doi:10.1016/j.ssci.2015 .06 .005 .

14. Ferster C, Fischer J, Manaugh K, Nelson T, Winters M. Using OpenStreetMap to inventory bicycle infrastructure: a comparison with open data from cities. Int J Sustain Transp 2020; 14(1):64-73. doi:10.1080/15568318 .2018 .1519746 .

15. Winters $M$, Teschke $K$, Brauer $M$, Fuller D. Bike Score ${ }^{\circledR}$ : associations between urban bikeability and cycling behavior in 24 cities. Int J Behav Nutr Phys Act. 2016;13(1):18. doi:10.1186 /s12966-016-0339-0.

16. Vijayakumar N, Burda C; Pembina Institute. Cycle cities: supporting cycling in Canadian cities. Toronto (Ont.): Pembina Institute; [mis à jour le 16 déc. 2015]. 